BUHEP-94-10

HUTP-94/A014

hep-ph/9406281

\title{
The Higgs Boson Width is Adjustable
}

\author{
R. Sekhar Chivukula ${ }^{a, 1}$ \\ Michael J. Dugan ${ }^{a, b, 2}$ \\ and \\ Mitchell Golden ${ }^{b, 3}$
}

\begin{abstract}
We show that it is possible to construct models in which the width of the Higgs boson is arbitrary - either smaller or larger than a standard model Higgs boson of the same mass. There are no new fields into which the Higgs boson decays. Instead, the coupling of the Higgs boson to the gauge bosons is adjusted. We construct and analyze weakly-coupled models with arbitrary-width Higgs bosons to investigate the phenomenology one might find in a strongly interacting model. In any such model new physics must enter at a mass scale which decreases as the Higgs boson width is adjusted away from its standard model value. In particular, if the Higgs boson is wider than the standard model Higgs boson, then interesting new physics must appear in the isospin-two channel.
\end{abstract}

$6 / 94$

\footnotetext{
${ }^{a}$ Boston University, Department of Physics, 590 Commonwealth Avenue, Boston, MA 02215

${ }^{b}$ Lyman Laboratory of Physics, Harvard University, Cambridge, MA 02138

${ }^{1}$ sekhar@abel.bu.edu

22dugan@physics.harvard.edu

${ }^{3}$ golden@physics.harvard.edu
} 


\section{Introduction}

In the standard model there exists a scalar particle with custodial isospin zero: the Higgs boson. There is a definite relationship between the Higgs boson's mass and its width to decay into gauge boson pairs. Suppose a custodial-isospin-zero scalar is discovered, but with a width which does not satisfy the standard model relationship. Though the particle content of the world appears to be the same as in the standard model, our hint that the standard model is not the whole story comes only from the unusual size of the coupling of the Higgs boson to other particles. The scalar particle with isospin zero in such a model has been termed a non-standard Higgs boson [1].

In this paper we construct models in which the Higgs boson is narrower or wider than a standard model Higgs boson of the same mass. We show that any theory with a non-standard Higgs particle must contain new physics at a mass scale which decreases as the couplings of the Higgs boson deviate from those of the standard model. We show that if the Higgs boson is wider than the standard model Higgs boson, and the model is perturbatively unitary, then the model must contain isospin-two resonances.

The models we discuss are only meant to be toy examples which contain a nonstandard Higgs boson. Such a Higgs particle could, for example, appear in a model with a strongly interacting symmetry breaking sector. This strongly interacting symmetry breaking sector, however, would have to be unlike conventional technicolor [2] which, in analogy to QCD, would be expected to contain only heavy and broad isospin-zero particles, i.e. particles having masses roughly the same as those of other technicolor resonances and widths comparable to their masses. The Higgs boson we consider in this paper is assumed to be light by comparison to any other resonances in the theory.

The plan of this paper is as follows. In the next section, we define notation and construct sample models that include Higgs bosons of arbitrary widths. In the third section we show that, if the Higgs width is larger than in the standard model then perturbative unitarity implies the existence of isospin-two resonances. In the fourth section we use the renormalization group and the triviality of theories with fundamental scalars to set an upper limit on the mass of the isospin-two multiplet in a model of fundamental scalars. This limit is substantially smaller than that given by the arguments of section three. In the fifth section we briefly discuss the radiative corrections to electroweak parameters in models with Higgs particles of non-standard widths. The last section states our conclusions. 


\section{How to Vary the Higgs Boson Width}

At sufficiently high energy the scattering of the longitudinally polarized $W$ and $Z$ bosons is approximately the same as that of the absorbed Goldstone bosons that would be present in the absence of the gauging of the electroweak symmetry. This statement is known as the "equivalence theorem" [3]. Such Goldstone bosons can conveniently be described in the language of chiral Lagrangians [4]. In our case, the Lagrangian contains the three "eaten" Goldstone bosons and a light isoscalar Higgs boson [1]:

$$
\mathcal{L}=\frac{1}{4}\left(v^{2}+2 \xi v H+\xi^{\prime} H^{2}+\ldots\right)\left(\operatorname{tr} \partial^{\mu} \Sigma^{\dagger} \partial_{\mu} \Sigma\right)+\ldots+\mathcal{L}_{H}
$$

where $\xi$ and $\xi^{\prime}$ are dimensionless coefficients, $v=246 \mathrm{GeV}$, and $\Sigma$ contains the swallowed Goldstone bosons $w^{a}$

$$
\Sigma=\exp \left(\frac{2 i \tau^{a} w^{a}}{v}\right) .
$$

Here $\tau^{a}$ are the generators of $S U(2)$, normalized to $\operatorname{tr} \tau^{a} \tau^{b}=\delta^{a b} / 2$. The first ellipsis indicates more terms coupling the Higgs boson to the Goldstone boson, (e.g. $H^{3},(\partial H)^{2}$, etc), and the second ellipsis indicates terms with more derivatives of the Goldstone bosons. These higher order terms are generically suppressed by some large scale, denoted $\Lambda_{\chi}$. We will assume nothing about $\Lambda_{\chi}$ except that it is large compared to the Higgs boson mass. In (2.1) $\mathcal{L}_{H}$ denotes the self-interaction Lagrangian for the Higgs boson

$$
\mathcal{L}_{H}=\frac{1}{2}(\partial H)^{2}-\frac{m_{H}^{2}}{2} H^{2}-\frac{\lambda_{3} v}{3 !} H^{3}-\frac{\lambda_{4}}{4 !} H^{4}-\ldots
$$

The standard model corresponds to the choice

$$
\xi=\xi^{\prime}=1, \lambda_{3}=\lambda_{4}=\frac{3 m_{H}^{2}}{v^{2}}
$$

with all higher order terms zero.

Using the lagrangian above, we may compute the width of the Higgs boson into $W W$ and $Z Z$. At tree-level, for Higgs bosons with mass much greater than twice the $Z$ mass, the width of the Higgs boson is

$$
\Gamma_{H}=\xi^{2} \frac{3 m_{H}^{3}}{32 \pi v^{2}} .
$$

Including the decay to quarks makes the Higgs boson only slightly broader, even for a relatively heavy top quark. In the standard model where $\xi=1$, there is a definite relationship between the Higgs boson's mass and its width. A measurement of the Higgs boson's width 
is a measurement of $\xi$. (The measurement of the parameters $\xi^{\prime}, \lambda_{3}$, and $\lambda_{4}$ is far more difficult than that of $\xi$. The Higgs boson's width is directly accessible to experiment; its self coupling is not [1].)

We will now see how the value of $\xi$ can be made different from 1, by considering models with fundamental scalars.

It is easy to see how one can make $\xi<1$. The strength of the coupling of the Higgs boson to the $W$ and $Z$ is dictated by the need to give the gauge bosons their observed mass. To reduce the coupling of the Higgs boson to the gauge bosons, one divides the vacuum expectation value among several fields. The field corresponding to a mass eigenstate Higgs boson does not get the full vacuum expectation value, $v$, and so it does not have the usual coupling to the gauge bosons. As an example, consider the two-Higgs-doublet model 1. The Lagrangian of the symmetry breaking sector is

$$
\mathcal{L}=\left(D^{\mu} \phi_{1}\right)^{\dagger}\left(D_{\mu} \phi_{1}\right)+\left(D^{\mu} \phi_{2}\right)^{\dagger}\left(D_{\mu} \phi_{2}\right)-V\left(\phi_{1}, \phi_{2}\right)
$$

where $V\left(\phi_{1}, \phi_{2}\right)$ is a quartic potential. The covariant derivative is

$$
D^{\mu} \phi_{i}=\left(\partial^{\mu}+i g W^{\mu} \cdot \tau-i \frac{g^{\prime}}{2} B^{\mu}\right) \phi_{i}
$$

If we put

$$
\phi_{i}=\left(\begin{array}{c}
\frac{H_{i}+v_{i}+i \phi_{i}^{0}}{\sqrt{2}} \\
i \phi_{i}^{-}
\end{array}\right)
$$

then the masses of the gauge bosons are, at tree level,

$$
M_{W}^{2}=\frac{g^{2}}{4}\left(v_{1}^{2}+v_{2}^{2}\right) \quad M_{Z}^{2}=\frac{g^{2}+g^{\prime 2}}{4}\left(v_{1}^{2}+v_{2}^{2}\right)
$$

so we conclude that $v_{1}^{2}+v_{2}^{2}=v^{2}$. It is customary to define $\beta$ such that $v_{1}=v \cos \beta$ and $v_{2}=v \sin \beta$. The spectrum of this model includes two Higgs bosons, a pair of charged pseudoscalars, and one neutral pseudoscalar. The mass-eigenstate Higgs bosons are a mixture of the fields $H_{1}$ and $H_{2}$. We write the lighter Higgs boson as

$$
H=-\sin \alpha H_{1}+\cos \alpha H_{2}
$$

The couplings of these particles to the gauge bosons may be worked out from (2.6). Let us imagine that the lighter of these two Higgs bosons is observed, but the heavier one eludes

1 For a review of Higgs boson properties in weakly-coupled theories, see [5]. 
experiment. The coefficient of the $H W^{a \mu} W_{\mu}^{a}$ term in the Lagrangian is $(1 / 4) g^{2}\left(-v_{1} \sin \alpha+\right.$ $\left.v_{2} \cos \alpha\right)$. The quantity $\xi$ is the ratio of this coupling to its value in the standard model, so

$$
\xi=\sin (\beta-\alpha) \quad, \quad \xi^{\prime}=1
$$

Therefore, $\xi^{2}<1$. This result is easily generalized to any number of doublets.

To get a Higgs boson with $\xi>1$ requires representations other than doublets. On the other hand, we do not want to spoil the successful tree level prediction of the ratio of the $W$ and $Z$ masses:

$$
\rho \equiv \frac{\left(g^{2}+g^{2}\right) M_{W}^{2}}{g^{2} M_{Z}^{2}}=1
$$

We are led to consider only models that have a "custodial" symmetry [6] [2]. This implies that the symmetry breaking sector must have a $S U(2)_{L} \times S U(2)_{R} \times U(1)_{V}$ symmetry 2 , with the hypercharge embedded in $S U(2)_{R} \times U(1)_{V}$.

Consider an $N \times N$ complex matrix field $\chi_{N}$ that transforms under $S U(2)_{L} \times S U(2)_{R}$ as

$$
\chi_{N} \rightarrow L \chi_{N} R^{\dagger}
$$

where the $L$ and $R$ are $S U(2)_{L}$ and $S U(2)_{R}$ group elements in the spin- $(N-1) / 2$ representation. To reduce the number of fields in the model, we impose the reality condition

$$
V \chi_{N} V^{\dagger}=\chi_{N}^{*}
$$

where $V$ is the $N \times N$ matrix connecting the spin- $(N-1) / 2$ representation to its complex conjugate. (For odd $N$, the representation is real, so $V$ can be chosen to be the identity.) We choose hypercharge to be generated by $T_{3}$ of $S U(2)_{R}$, and thus

$$
D^{\mu} \chi_{N}=\partial^{\mu} \chi_{N}+i g W^{\mu} \cdot T \chi_{N}-i g^{\prime} \chi_{N} B^{\mu} T_{3}
$$

where $T^{a}$ are the generators of $S U(2)$ in the spin- $(N-1) / 2$ representation. (This is a generalization of the models considered in [7].) The kinetic energy term in the Lagrangian is

$$
\mathcal{L}_{K E}=\frac{1}{2} \operatorname{tr}\left(\left(D \chi_{N}\right)^{\dagger}\left(D \chi_{N}\right)\right)
$$

2 Actually, the lagrangian (2.1) written above imposed such a symmetry too. With only the $S U(2)_{W} \times U(1)_{Y}$ symmetry terms such as $\operatorname{tr} \partial^{\mu} \Sigma^{\dagger} \partial_{\mu} \Sigma T_{3}$ are not forbidden. 
In order to preserve the relation (2.12), we assume that the potential for this field gives it a vev proportional to the identity. There is one isosinglet field $H$ in $\chi_{N}$ and we put

$$
\chi_{N}=\frac{H+v_{N}}{\sqrt{N}} I+\ldots .
$$

Now the interactions among the gauge and Higgs boson are of the form

$$
\mathcal{L}_{K E}=\frac{1}{2} \frac{v_{N}^{2}}{N}\left(1+\frac{H}{v_{N}}\right)^{2}\left(\operatorname{tr}\left(T_{a} T_{b}\right) g^{2} W_{a} W_{b}+\operatorname{tr}\left(T_{3}^{2}\right)\left(2 g g^{\prime} W_{3} B+g^{\prime 2} B^{2}\right)\right)+\ldots
$$

The $W$ and $Z$ get masses in the correct ratio. Using $\operatorname{tr}\left(T_{a} T_{b}\right)=N\left(N^{2}-1\right) / 12$, we find

$$
M_{W}^{2}=g^{2} \frac{N^{2}-1}{12} v_{N}^{2}
$$

From (2.18) we conclude

$$
\xi=\frac{v}{v_{N}}=\sqrt{\frac{N^{2}-1}{3}} .
$$

Thus we see that the value of $\xi$ can be made as large as we like.

To conclude this section we point out that it is possible to get values of $\xi$ other than those of (2.20) by mixing Higgs bosons from different representations. If two Higgs bosons with respective couplings to $W$ pairs parameterized by $\xi_{1}$ and $\xi_{2}$ mix by an angle $\alpha$, then the value of $\xi$ for the lighter of the two Higgs bosons is $\xi=-\xi_{1} \sin \alpha+\xi_{2} \cos \alpha$.

\section{Unitarity of Goldstone Boson Scattering}

Let us suppose that an experiment has seen a Higgs boson with width parameterized by $\xi$. At what scale need new physics enter, and what form can it take?

The most general form of the scattering amplitude of the Goldstone bosons $w^{a}$ consistent with crossing, Bose symmetry, and custodial isospin invariance is

$$
a\left(w^{a} w^{b} \rightarrow w^{c} w^{d}\right)=A(s, t, u) \delta^{a b} \delta^{c d}+A(t, s, u) \delta^{a c} \delta^{b d}+A(u, s, t) \delta^{a d} \delta^{b c}
$$

where $A$ is a function of the three Mandelstam variables symmetric in its last two arguments. The Lagrangian (2.1) contains two Feynman diagrams that contribute to $A$ at lowest order in $\Lambda_{\chi}$ : one contact diagram and one in which the Higgs boson is exchanged in the $s$-channel. Their sum is

$$
A(s, t, u)=\frac{s}{v^{2}}\left(1-\frac{s \xi^{2}}{s-m_{H}^{2}}\right) .
$$


In the standard model in which $\xi=1$, there is at high energies a cancellation between the first and second terms. The growth with $s$ of the amplitude is truncated by the appearance of the Higgs boson. An argument of Lee, Quigg, and Thacker [8] shows that if the standard model is to be unitary at tree-level at high energies, i.e. if the absolute value of all scattering amplitudes of all partial waves is to remain less than 1 , then the mass of the Higgs boson must be less than about $1 \mathrm{TeV}$.

If $\xi \neq 1$, the absolute value of the amplitude continues to grow with $s$ even above the Higgs boson mass. If we assume, following Lee, Quigg, and Thacker, that the amplitude is to be unitarized by the exchange of spin-zero resonances, then we see that there are two possibilities: resonances of isospin zero or two. No other scalars can couple to a pair of Goldstone bosons.

The contribution of another isospin-zero scalar is like that of the lightest Higgs boson, so it makes a negative contribution to the amplitude at high energies. If all the resonances are isospin zero the requirement that the amplitude stop growing at large $s$ implies 9

$$
\sum_{i} \xi_{i}^{2}=1
$$

where $\xi_{i}$ is defined for each resonance. Clearly, if the model contains a Higgs boson with $\xi>1$, there must be something other than isosinglets to unitarize the amplitude.

An isospin-two multiplet, by contrast, is exchanged in the $s, t$, and $u$ channels. If the multiplet is represented by a symmetric traceless matrix $S^{a b}$, it may be included into the chiral Lagrangian by adding

$$
\mathcal{L}_{S}=\frac{1}{4}\left(\partial^{\mu} S^{a b}\right)\left(\partial_{\mu} S^{a b}\right)+\frac{C}{v} S^{a b} \partial^{\mu} \pi_{a} \partial_{\mu} \pi_{b}+\ldots
$$

where $C$ is a dimensionless coupling constant. This field makes the contribution

$$
\frac{C^{2}}{v^{2}}\left[\frac{2}{3} \frac{s^{2}}{s-m_{2}^{2}}-\left(\frac{t^{2}}{t-m_{2}^{2}}+\frac{u^{2}}{u-m_{2}^{2}}\right)\right]
$$

to $A(s, t, u)$, where $m_{2}$ is the mass of the isospin-two representation. Note that at high energies, where $s, t$, and $u$ are much greater than $m_{2}^{2}$, the net contribution of the isospintwo multiplet to $A$ is

$$
\frac{5 C^{2} s}{3 v^{2}}
$$

a positive quantity. 
If, as before, we insist that the amplitude stops growing at high energies, there will be a sum rule connecting the couplings of the isosinglet Higgs bosons to those of the isospin-two multiplets 3

$$
\sum_{i} \xi_{i}^{2}=1+\frac{5}{3} \sum_{j} C_{j}^{2}
$$

Here $i$ runs over the Higgs bosons and $j$ runs over the isospin-two multiplets. Therefore, if a model has a Higgs boson with $\xi>1$ and is unitarized by the exchange of a spin-zero resonance, it must contain at least one isospin-two representation.

Consider the example given in the last section, in which the symmetry breaking sector was an $N \times N$ matrix of fundamental scalars. After the symmetry breaks, the mass eigenstates fall into degenerate multiplets of custodial isospin - one multiplet each of custodial isospin from $N-1$ down to zero. The three isospin-one particles are the absorbed degrees of freedom, but the remaining particles are physical. For $N>2$ there is an isospintwo multiplet and $C$ can be computed explicitly,

$$
C^{2}=\frac{\left(N^{2}-4\right)}{5}
$$

With the parameter $\xi$ is given in (2.20), the sum rule (3.7) is satisfied.

The Lee, Quigg, and Thacker argument gives an upper limit on the mass-scale of new physics, $M$. For $m_{H}^{2} \ll s \ll M^{2}$, the amplitude is growing in magnitude

$$
A(s, t, u) \sim\left(1-\xi^{2}\right) \frac{s}{v^{2}}
$$

Since the isospin-zero, spin-zero amplitude

$$
a_{00}(s)=\left(1-\xi^{2}\right) \frac{s}{16 \pi v^{2}}
$$

must be bounded in magnitude by one, we conclude that

$$
M^{2} \leq \frac{16 \pi v^{2}}{\left|\xi^{2}-1\right|}=\frac{(1750 \mathrm{GeV})^{2}}{\left|\xi^{2}-1\right|}
$$

In the $N \times N$ matrix models, where $\xi>1$, eqn. (3.11) bounds the mass of the isospin-two resonance. In a two-doublet Higgs model, the eqn. (3.11) gives a bound on the mass of the heavier Higgs boson.

3 This is a special case of the sum rules derived in 10 . 
The argument of this section shows that as the parameter $\xi$ is changed away from its standard model value, the scale of new physics is reduced. Even in a strongly interacting model, which will not in general be unitarized by perturbative physics (and so the sum rule (3.7) might not be valid, or the isospin-two particles might even be absent), this qualitative feature of (3.11) should continue to hold. There is a maximum scale at which some new physics must enter, and that scale is reduced as the non-standard Higgs boson looks less like the standard model Higgs boson.

\section{Renormalization Group Analysis}

For the scalar model with $N=3$, one finds $\xi^{2}=8 / 3$ and the Lee, Quigg, and Thacker bound (3.11) on $m_{2}$ is approximately $1400 \mathrm{GeV}$. In this section we will use the renormalization group and the triviality of theories with fundamental scalars to set an upper limit on the mass of the isospin-two multiplet in this model that is substantially smaller.

Suppose one Higgs boson with $\xi^{2}=8 / 3$ and isospin-two scalars with $C^{2}=1$ are discovered. If there is a large hierarchy between the masses of these particles and the masses of other new physics (such as compositeness effects in the scalars), the model must be approximately renormalizable. This world therefore appears to be described by the model with $\chi_{3}$, the $3 \times 3$ matrix [7]. On the other hand, any model with fundamental scalars is trivial, and thus despite its renormalizability the model does not make sense above a certain energy scale. We must set the scale of new physics below that point. We may compute the $\beta$-function of this model and integrate to find the Landau pole. For the isospin-two bosons to be approximately fundamental, we must insist that their mass be smaller than some factor times the mass of the Landau pole.t

The potential for the $3 \times 3$ model is

$$
V(\chi)=g_{1}\left(\operatorname{tr} \chi_{3}^{t} \chi_{3}-v_{3}^{2}\right)^{2}+g_{2}\left[3 \operatorname{tr} \chi_{3}^{t} \chi_{3} \chi_{3}^{t} \chi_{3}-\left(\operatorname{tr} \chi_{3}^{t} \chi_{3}\right)^{2}\right]
$$

4 This is analogous to the Dashen-Neuberger bound for the standard model [11]. A recent review of lattice simulations and analytic studies of the Higgs sector of the standard model [12] reports that $m_{H}$ must be less than about $700 \mathrm{GeV}$. 
The minimum of this potential is at $v_{3} I$ if and only if the dimensionless constants $g_{1}$ and $g_{2}$ are positive. It is the $N=3$ case of a model with an $O(N)_{L} \times O(N)_{R}$ symmetry, the $\beta$-functions for which are [13]

$$
\begin{aligned}
& 2 \pi^{2} \mu \frac{\partial}{\partial \mu} g_{1}=\left(N^{2}+8\right) g_{1}^{2}+\left(2 N^{2}+2 N-4\right) g_{1} g_{2}+2\left(N^{2}+2 N-4\right) g_{2}^{2} \\
& 2 \pi^{2} \mu \frac{\partial}{\partial \mu} g_{2}=12 g_{1} g_{2}+2\left(N^{2}+2 N-6\right) g_{2}^{2} .
\end{aligned}
$$

This potential gives mass to the Higgs boson and the isospin-two particles. The masses at tree level are

$$
m_{H}^{2}=8 g_{1} v_{3}^{2} \quad, \quad m_{2}^{2}=8 g_{2} v_{3}^{2}
$$

We now integrate the $\beta$-functions to find the Landau pole. We define $t=\log \left(\mu_{P} / \mu\right)$, where $\mu_{P}$ is the value of $\mu$ at the Landau pole. The form of the differential equations is such that both $g_{1}$ and $g_{2}$ have a pole at the same value of $\mu$. The pole in $g_{1}$ goes like $1 / t$, while the pole in $g_{2}$ goes like

$$
g_{2} \sim\left(\frac{1}{t}\right)^{\frac{12}{\left(N^{2}+8\right)}}
$$

With this information we define the variables

$$
x_{1}=\frac{1}{g_{1}}, \quad x_{2}=\left(\frac{1}{g_{2}}\right)^{\frac{\left(N^{2}+8\right)}{12}}
$$

and integrate the equations for them.

In fig. 11 we show the set of points in $g_{1}, g_{2}$ space with $t=1$. This is the set of coupling constants $g_{1}(\mu), g_{2}(\mu)$, such that the Landau pole is at a mass of $e \mu$ (where $e$ the base of the natural logarithm). The equations (4.2) are such that if one performs a transformation

$$
g_{1} \rightarrow K g_{1} \quad, \quad g_{2} \rightarrow K g_{2} \quad, \quad t \rightarrow \frac{t}{K}
$$

for any positive $K$, then the result is still a solution. Thus, if we know where the $t=1$ contour is in $g_{1}, g_{2}$ space, we know where all the other contours are.

In fig. 1 the value of $g_{2}$ is never bigger than about .75. Since the mass of the isospintwo multiplet is

$$
m_{2}^{2}=8 g_{2}\left(m_{2}^{2}\right) v_{3}^{2}=3 g_{2}\left(m_{2}^{2}\right) v^{2}
$$

we deduce that the model does not make sense if $m_{2}$ is more than about $375 \mathrm{GeV}$ ! This upper limit applies at the small $g_{1}$ end of the contour, where the Higgs boson is light. As the Higgs boson gets heavier, the limit decreases. 
In fundamental scalar theories with $N$ larger than 3 or with scalars in other representations, these limits only get stronger: the extra fields give additional positive contributions to the $\beta$-functions, leading to lower values for the Landau pole for a given value of $g_{1}$ and $g_{2}$. We conclude that in models of fundamental scalars with $\xi>1$, the isospin-two multiplet must be fairly light.

\section{Radiative Corrections}

We have seen that a wide Higgs boson implies the existence of new physics at a relatively low scale. The expectation is that the new physics makes a contribution to electroweak radiative parameters, which we parameterize in terms of $S, T$, and $U$ [14]

Consider first the example of the $3 \times 3$ matrix $\chi_{3}$ [7]. In this model, $S$ is calculable. The loops of isospin-two particles give a new contribution.

$$
S=-\frac{5}{36 \pi}\left[\frac{5}{2}-\log \left(\frac{m_{H}^{2}}{m_{2}^{2}}\right)\right]+\frac{1}{12 \pi} \log \left(\frac{m_{H}^{2}}{1 \mathrm{TeV}^{2}}\right)
$$

This is a small negative number whenever $m_{H}<m_{2}<1 \mathrm{TeV}$.

It is easy to see, however, that the corrections to $T$ and $U$ are not calculable in this model 5 . Electroweak gauge invariance permits the addition of custodial symmetry violating terms to the Lagrangian

$$
\begin{aligned}
\mathcal{L}_{\text {new }}= & a_{1} \operatorname{tr}\left[\left(D^{\mu} \chi_{3}\right)^{t}\left(D^{\mu} \chi_{3}\right) T_{3}^{2}\right]+a_{2} v_{3}^{2} \operatorname{tr}\left(\chi_{3}^{t} \chi_{3} T_{3}^{2}\right)+a_{3} \operatorname{tr}\left(\chi_{3}^{t} \chi_{3} \chi_{3}^{t} \chi_{3} T_{3}^{2}\right) \\
& +a_{4} \operatorname{tr}\left(\chi_{3}^{t} \chi_{3} T_{3} \chi_{3}^{t} \chi_{3} T_{3}\right)+a_{5}\left|\operatorname{tr}\left(\chi_{3}^{t} \chi_{3}\left(T_{1}+i T_{2}\right)^{2}\right)\right|^{2}
\end{aligned}
$$

In fact, the addition of these terms to the Lagrangian is actually required. The gauging of only one of the three generators of the $S U(2)_{R}$ breaks the custodial symmetry, and these terms are required as counterterms for diagrams involving the hypercharge gauge boson. The $a_{2}, a_{3}, a_{4}$, and $a_{5}$ terms cause the vev of the $(3,3)$ entry of $\chi_{3}$ to be different from the $(1,1)$ and $(2,2)$ entries, splitting the tree-level mass of the $W$ and $Z$. The $a_{1}$ term directly shifts the mass of the gauge bosons. The situation is precisely analogous to the splitting between the $\pi^{ \pm}$and $\pi^{0}$ masses generated by photon loops. While these coefficients are not

5 Corrections to $T$ in this model are discussed in detail in [15]. Our point of view is somewhat different. In [15], it was assumed that the Higgs and isospin-two particles are fundamental, and the one-loop computations determined the degree of fine-tuning needed to make $T$ small enough. We regard the particles as composite, and instead seek to limit the scale of their compositeness. 
formally computable, one may estimate them by dimensional analysis. One expects that the renormalized value of $a_{2}$ is of order

$$
a_{2} \sim \frac{g^{\prime 2}}{16 \pi^{2}} \frac{\Lambda^{2}}{v_{3}^{2}}
$$

where $\Lambda$ is the cutoff of the theory. The $a_{1}, a_{3}$, and $a_{4}$ terms depend only logarithmically on $\Lambda$. The $a_{2}$ term leads to

$$
T=\frac{2 a_{2} v_{3}^{2}}{\alpha m_{2}^{2}} \sim \frac{1}{2 \pi \cos ^{2} \theta_{W}} \frac{\Lambda^{2}}{m_{2}^{2}} .
$$

Since one expects new physics at the scale of the cutoff, if such a model is to be phenomenologically acceptable, the mass scale of the new physics is only about a factor of two heavier than the mass of the isospin-two resonances.

Another possibility is that the isospin-two scalars do not exist as approximately fundamental particles, and in that case one must continue to use the chiral Lagrangian (2.1) up to $\Lambda_{\chi}$, where some unspecified new physics appears. Here, again, loops containing the $B$ gauge boson require the addition of counterterms that do not respect the custodial symmetry. Once again, $T$ and $U$ are not calculable, but there is a significant difference. There is no custodial-symmetry-violating dimension-two term in the low energy theory, and thus the corrections to $T$ are not enhanced by a factor of $\Lambda^{2} / v_{3}^{2}$. Unlike the scalar case, $S$ is not calculable either, due to the presence of counterterms like [16]

$$
\mathcal{L}_{S}=\operatorname{tr}\left(\left(\left[D^{\mu}, D^{\nu}\right] \Sigma\right)^{\dagger}\left[D^{\mu}, D^{\nu}\right] \Sigma\right)
$$

As in any strongly interacting theory, one needs information about the physics at high energies to say more about the radiative parameters.

\section{Conclusions}

We have seen that it is possible to construct models in which the Higgs boson has any desired width, either larger or smaller than in the standard model. The less the Higgs boson looks like its standard model counterpart, the lower the mass scale at which new physics must enter. If the theory is tree-level unitary at high energies and the Higgs boson width is greater than in the standard model, there must be isospin-two resonances. Furthermore, if these isospin-two particles are to be considered approximately fundamental they must 
be light. One needs information about the physics at high energies to be definite about the sizes of electroweak radiative corrections, but they are expected to be significant.

Work on the production and detection of such a non-standard Higgs boson is in progress [17].

\section{Acknowledgments}

We would like to thank Dimitris Kominis, Vassilis Koulovassilopoulos, Ken Lane, and Elizabeth Simmons for discussions and for comments on the manuscript. R.S.C. acknowledges the support of an Alfred P. Sloan Foundation Fellowship, an NSF Presidential Young Investigator Award, and a DOE Outstanding Junior Investigator Award. M.G. acknowledges the support of an NSF National Young Investigator Award. This work was supported in part under NSF contracts PHY-9218167 and PHY-9057173 and DOE contract DE-FG02-91ER40676, and by funds from the Texas National Research Laboratory Commission under grants RGFY93-278 and RGFY93-278B. 


\section{References}

[1] R. S. Chivukula, V. Koulovassilopoulos, Phys. Lett. B 309, 371 (1993) ;

V. Koulovassilopoulos, R. S. Chivukula, Boston University Preprint BUHEP-93-30, Dec 1993, hep-ph - 9312317.

[2] S. Weinberg, Phys. Rev. D 19, 1979 (1277);

L. Susskind, Phys. Rev. D 20, 1979 (2619).

[3] J. Cornwall, D. Levin, and G. Tiktopoulos, Phys. Rev. D 10, 1145 (1974);

C. Vayonakis, Lett. Nuovo Cimento 17, 383 (1976);

M. S. Chanowitz and M. K .Gaillard, Nucl. Phys. B 261, 379 (1985).

[4] S. Coleman, J. Wess, and B. Zumino, Phys. Rev. 177, 2239 (1969);

C. Callan, S. Coleman, J. Wess, and B. Zumino, Phys. Rev. 177, 2247 (1969).

[5] The Higgs Hunter's Guide, J. F. Gunion, et. al., Addison Wesley, New York, 1990.

[6] M. Weinstein, Phys. Rev. D8, 2511 (1973);

see also P. Sikivie, L. Susskind, M. Voloshin, and V. Zakharov, Nucl. Phys. B. 173 (1980) 189.

[7] P. Galison Nucl. Phys. B 232, 26 (1984);

H. M. Georgi and M. Machacek, Nucl. Phys. B 262, 463 (1985);

M. S. Chanowitz and M. Golden, Phys. Lett. B 165, 105 (1985).

[8] B. W. Lee, C. Quigg, and H. B. Thacker, Phys. Rev. D16, 1519 (1977).

[9] J. F. Gunion, R. Vega, and J. Wudka, Phys. Rev. D 42, 1673 (1990)

[10] J. F. Gunion, H. E. Haber, and J. Wudka, Phys. Rev. D 43, 904 (1991).

[11] R. Dashen and H. Neuberger, Phys. Rev. Lett. 50, 1897 (1983).

[12] U. M. Heller, M. Klomfass, H. Neuberger, and P. Vranas, Nucl. Phys. B 405, 555 (1993).

[13] R. D. Pisarski and D. L. Stein, Phys. Rev. B. 23, 3549 (1981) and J. Phys. A. 14, 3341 (1981).

[14] M. E. Peskin and T. Takeuchi, Phys. Rev. D46, 381 (1992).

[15] J. Gunion, R. Vega, and J. Wudka, Phys. Rev. D 43, 2322 (1991).

[16] T. Appelquist, lectures presented at the 21st Scottish Universities Summer School in Physics, St. Andrews, Scotland, Aug 10-30, 1980;

A. C. Longhitano, Nucl. Phys. B 188, 118 (1981);

M. Golden and L. Randall, Nucl. Phys. B 361, 3 (1991);

B. Holdom and J. Terning, Phys. Lett. B 247, 88 (1990);

A. Dobado, D. Espriu, and M. Herrero, Phys. Lett. B 253, 161 (1991).

[17] D. Kominis and V. Koulovassilopoulos, in preparation. 


\section{Figure Captions}

Fig. 1. The set of points in $g_{1}, g_{2}$ space with $t=1$. 


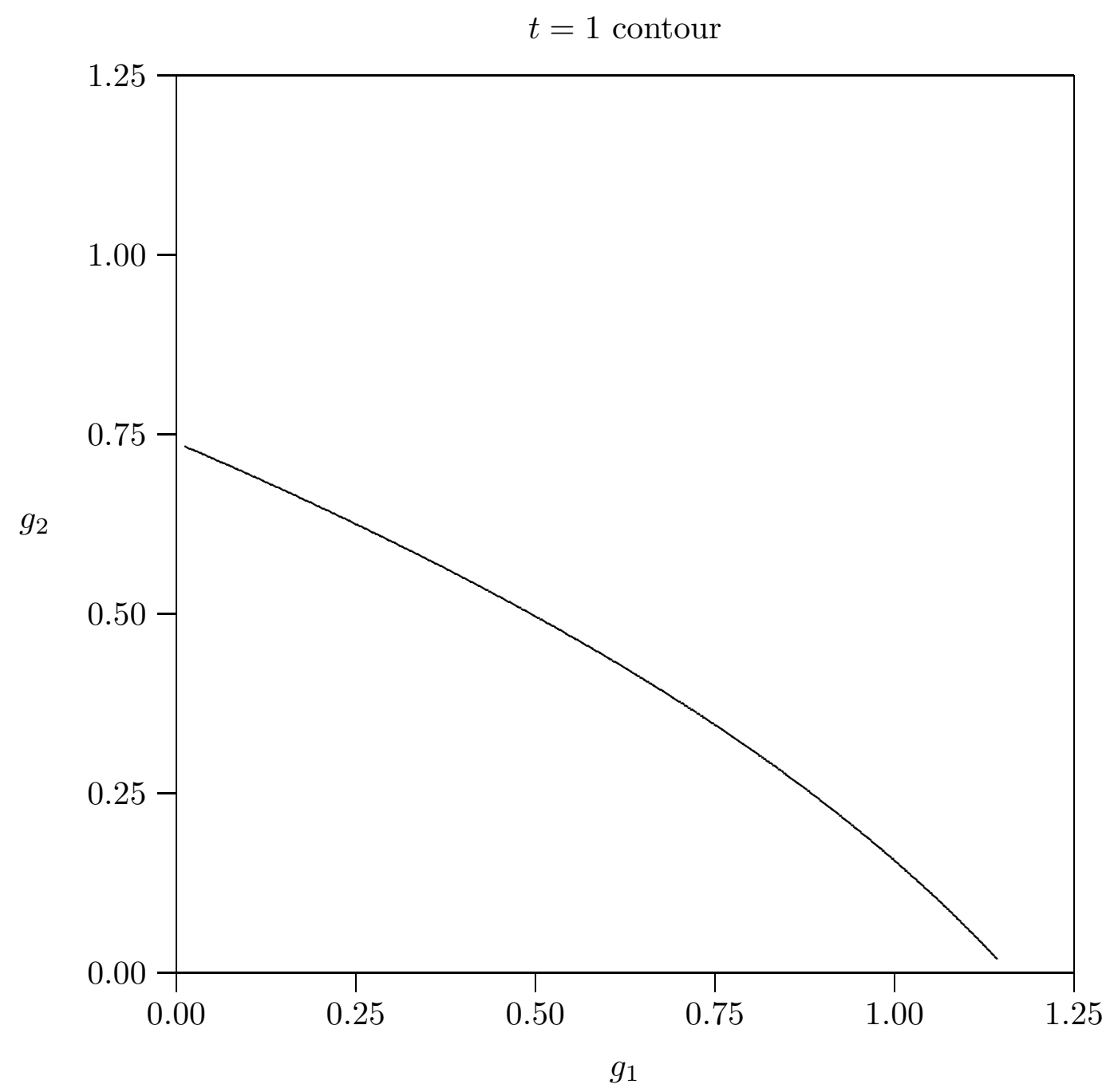

